\title{
Synthesis and characterization of grafted polystyrene with acrylonitrile using gamma- irradiation
}

\begin{abstract}
Polystyrene grafted with acrylonitrile using gamma-irradiation technique was successfully synthesized. This process was carried out at various gamma dose (0.2-1.5 Mrad). The new grafted polymer was characterized and its properties were investigated. The results indicate that best grafting percentage ratio could be revealed when the concentration of the catalyst Ferrous Ammonium Sulphate (FAS) is about $2 \%$ and the monomer concentration is $90 \%$, at 1.25 Mrad dose. The new grafted polymer was proved by FTIR, TGA and Viscosity techniques, which was analyzed and studied with a suggested presented mechanism. The adding of acrylonitrile to polystyrene improved the physical properties of polystyrene
\end{abstract}

Keyword: Polystyrene; Acrylonitrile; FAS; Gamma-irradiation; Grafted Polymer. 